\title{
The Prophylactic Effect of Proton Pump Inhibitors Combined with Enteral Nutrition for Preventing Gastrointestinal Hemorrhage after Cardiovascular Surgery in High-Risk Patients
}

\author{
Mei-Fang Chen, MD, Yong Lin, MM, Liang-Wan Chen, MD \\ Department of Cardiovascular Surgery, Fujian Medical University Union Hospital, Fuzhou, Fujian Province, China
}

\section{ABSTRACT}

Background: Gastrointestinal hemorrhage (GH) is one of the most serious complications after cardiovascular surgery. The aim of the study was to provide an optimal therapeutic strategy for preventing postoperative GH in high-risk patients. Methods: This retrospective case-control study included 188 adult patients at high risk of postoperative GH. These patients were divided into two groups based on a strategy for preventing postoperative GH: Group A $(n=97)$ received continuous intravenous infusion of protonpump inhibitor (PPI) combined with early enteral nutrition, and Group B ( $\mathrm{n}=91)$ received a bolus intravenous infusion of PPI combined with late enteral nutrition. The clinical features of the groups were examined.

Results: The incidence of postoperative GH in the patients of group A was significantly lower than the patients in group B. The duration from the end of surgery to eating for the first time in the patients of group A was significantly shorter than in the patients of group B. A descending trend in 30-day mortality was observed in the patients of group A compared with group B, but no significant difference was found between the two groups.

Conclusion: Continuous intravenous infusion of PPI combined with early enteral nutrition could effectively prevent GH and reduce 30-day mortality after cardiovascular surgery in high-risk patients.

\section{INTRODUCTION}

Gastrointestinal hemorrhage is rare (0.3\%-1.0\%) but often fatal (8.8\%-47.6\%) [Andersson 2005; Rodriguez 2010; D’Ancona 2003; Krawiec 2017; Fan 2010; Chaudhry 2017] and is one of the most serious complications after cardiovascular surgery; its occurrence noticeably prolongs the duration of hospitalization and intensive care unit (ICU) stays and increases hospital costs and the rate of transfusion [D'Ancona 2003; Grus 2014; Ait Houssa 2007]. Gastrointestinal

Received November 5, 2018; accepted December 20, 2018.

Liang-Wan Chen, Department of Cardiovascular Surgery, Fujian Medical University Union Hospital, Fuzhou, Fujian Province, PR China; (e-mail: birdman1983@163.com). hemorrhage has been reported to be induced by surgical stress, imbalance of intestinal flora, ischemic necrosis of gastrointestinal mucosa and perioperative inflammation [Ohri 2006; Quenot 2009; Perugini 1997]. Prophylactic administration of proton pump inhibitors (PPI) or antacid agents that protect the gastrointestinal mucosal barrier have been considered to be the most important strategy to prevent postoperative gastrointestinal hemorrhage [Patel 2013]. However, some postoperative patients who received the recommended prophylactic strategy were still found to suffer from gastrointestinal hemorrhage, and some of them died from it. Therefore, it is very important to find a more effective multimodality prophylactic strategy of gastrointestinal hemorrhage that will improve the prognosis of patients after cardiovascular surgery.

\section{METHODS}

This single-center retrospective clinical case-control study included 188 high-risk patients who underwent cardiovascular surgeries in the Department of Cardiovascular Surgery, Fujian Medical University Union Hospital, between January 2017 and December 2017. The Ethics Committees of Fujian Medical University Union Hospital approved this retrospective study.

These patients were divided into two groups according to the prophylactic strategy for addressing gastrointestinal hemorrhage: Group A ( $\mathrm{n}=97)$ received a continuous intravenous infusion of PPI combined with early enteral nutrition, and Group B $(n=91)$ received a bolus intravenous infusion of PPI combined with late enteral nutrition (Figure 1). Omeprazole Sodium for Injection (Losec, $40 \mathrm{mg}$, AstraZeneca) was the PPI used for both groups. Patients in group A were administered PPI through continuous intravenous injection $80 \mathrm{mg}$ qd for 3-7 days or more days, if the pathogenesis of the condition required it after surgery. Probiotics and enteral nutrition beginning with clear liquids and followed by oligopeptide and dietary fiber were provided to these patients by nasogastric tube or oral administration within 12-24 h after surgery, regardless of extubation. The patients lay in a supine position with 30 degrees of elevation and walked early. The dosage of vasoactive drugs was reduced as soon as possible if pathogenesis conditions permitted. Patients in group B were administered PPI through a bolus intravenous injection $40 \mathrm{mg}$ bid for 3-7 days or more days, if pathogenetic condition required 
it and were fed through a nasogastric tube or by oral administration within $48 \mathrm{~h}$ after surgery or within $6 \mathrm{~h}$ after extubation. The remainder of the prophylactic protocols were similar to those used for the patients in the group A.

\section{Timing of PPI Withdrawal}

PPI was withdrawn under the following conditions: (1) The hemodynamics of the patients were stable after extubation and they could eat normally or be fed with enteral nutrition through a nasogastric tube without gastrointestinal symptoms such as abdominal pain, ventosityor dysphoria; (2) Death occurred during therapeutic process; (3) Adverse drugs reactions were detected that were intolerant and suspected to be associated with PPI.

\section{Inclusion Criteria}

Adult patients who underwent cardiovascular surgery with cardiopulmonary bypass (CPB) and had at least one of the high-risk factors mentioned in the gastrointestinal complications after cardiac surgery (GICS) score [Andersson 2010].

\section{Exclusion Criteria}

(1) Patients with active gastrointestinal bleeding within 4 weeks before surgery; (2) Patients with esophageal and gastric varices associated with preoperative cirrhosis with portal hypertension; (3) Patients with hereditary or acquired coagulopathy; (4) Patients with hemorrhage from hemorrhoid; (5) Patients with gastrointestinal bleeding from intestinal ischemic necrosis caused by mesenteric artery embolism; (6) Patients who died within $24 \mathrm{~h}$ after surgeries.

\section{Diagnosis of Gastrointestinal Hemorrhage}

(1) Patients with visible hematemesis or melena confirmed by the results of an OB test; (2) Patients with instable hemodynamics and a reduction in the concentration of hemoglobin in the peripheral blood; (3) Patients with a positive result from an $\mathrm{OB}$ test of the drainage of digestive juices, vomit or feces; (4) Patients with an active hemorrhagic focus (excluding Dieulafoy's disease or gastrointestinal tumor) on the surface of gastrointestinal mucosa, confirmed by endoscopic examination or digital subtraction angiography (DSA); (5) Patients with bleeding in the respiratory tract.

\section{General Clinical Data}

General clinical data included age, sex, body mass index (BMI), history of use of anticoagulants or antiplatelet agents, coagulation function, the Child-Pughscore, New York Heart Association (NYHA) class, underlying diseases including history of gastrointestinal disease, hypertension, diabetes, hyperlipidemia, coronary artery disease (CAD), stroke, active smoking, alcoholism, GICS score, and EuroSCORE II.

\section{Surgical and Intraoperative Treatments}

Surgical and intraoperative treatments included type of surgical correction, duration of surgery, cardiopulmonary bypass $(\mathrm{CPB})$, aortic cross-clamping, hypothermic circulatory arrest (HCA) and thoracic closure, and intraoperative blood loss.

\section{Laboratory Examination}

The laboratory findings used were the worst results of the perioperative routine blood test, conventional coagulation function test and thromboelastography (TEG).

\section{Postoperative Statistics}

The postoperative conditions assessed included length from the end of the operation to eating for the first time, the length of time from the end of the operation to defecation for the first time, meteorism, nausea, vomiting, abdominal pain, diarrhea, constipation, headache, the result of the fecal $\mathrm{OB}$ test, the volume of postoperative thoracic drainage, the volume of the allogenetic transfusion including red blood cells, fresh frozen plasma, platelets and cryoprecipitation.

\section{Pharmacotberapy}

Medications assessed included vasoactive agents, anticoagulants, antiplatelet agents and high-dose glucocorticoids.

\section{Mechanical Assistance}

Mechanical assistance assessed included the application of an intraaortic balloon pump (IABP) or/and extracorporeal membrane oxygenation (ECMO).

\section{Short-term Prognosis}

The short-term prognosis considered 30-day postoperative mortality, the duration of postoperative mechanical ventilation, ICU stays and hospitalization, postoperative complications including low cardiac output syndrome (LCOT), renal dysfunction, hepatic dysfunction, sepsis, multiple organ dysfunction syndrome (MODS), acute respiratory distress syndrome (ARDS), pulmonary infection, stroke, hypoproteinemia, reoperation for bleeding, and hyperlactacidemia. The follow-up reviews were conducted every 6 months after discharge. In cases of death after discharge, the cause was ascertained with the relatives' permission.

\section{Statistical Analysis}

SPSS 19.0 software was used for statistical analyses. Descriptive statistical analyses, Wilcoxon rank sum tests and repeated-measures ANOVAs were used to analyze measurement data. The Pearson chi-square test and Fisher exact test were used to analyze the count data. The Kaplan-Meier method was used to plot the survival curves. Statistical significance was defined as $P<.05$.

\section{RESULTS}

\section{General Clinical Data}

In total, 188 adult patients at high risk of gastrointestinal hemorrhage after cardiovascular surgery were included in this retrospective study, including 126 cases of cardiac valvular disease (CVD), 32 cases of CAD, 17 cases of acute aortic dissection (AAD), 10 cases of congenital heart disease (CHD) and 3 cases of heart transplantation (HT) (Table 1). 
Table 1. Clinical Data

\begin{tabular}{|c|c|c|c|}
\hline & $\begin{array}{l}\text { Group A } \\
(n=97)\end{array}$ & $\begin{array}{l}\text { Group B } \\
(n=91)\end{array}$ & $P$ \\
\hline \multicolumn{4}{|l|}{ Protopathy, n (\%) } \\
\hline CVD & $64(66.0)$ & $62(68.1)$ & .754 \\
\hline CAD & $18(18.6)$ & $14(15.4)$ & .563 \\
\hline AAD & $9(9.3)$ & $8(8.8)$ & .907 \\
\hline $\mathrm{CHD}$ & $4(4.1)$ & $6(6.6)$ & .450 \\
\hline $\mathrm{HT}$ & $2(2.1)$ & $1(1.1)$ & 1.000 \\
\hline Age & $48.2 \pm 12.4$ & $49.7 \pm 9.9$ & .298 \\
\hline \multicolumn{4}{|l|}{ Sex, n (\%) } \\
\hline Male & $69(71.1)$ & $63(69.2)$ & .776 \\
\hline Female & $28(28.9)$ & $28(30.8)$ & \\
\hline BMI & $21.8 \pm 4.2$ & $22.3 \pm 3.8$ & .721 \\
\hline \multicolumn{4}{|l|}{ Pharmacotherapy, n (\%) } \\
\hline Anticoagulant drugs & $8(8.2)$ & $5(5.5)$ & .457 \\
\hline Antiplatelet drugs & $21(21.6)$ & $18(19.8)$ & .752 \\
\hline \multicolumn{4}{|l|}{ Child-Pugh score, n (\%) } \\
\hline 1 & $83(85.6)$ & $79(86.8)$ & 1.000 \\
\hline 2 & $13(13.4)$ & $12(13.2)$ & \\
\hline 3 & $1(1.0)$ & $0(0)$ & \\
\hline 4 & $0(0)$ & $0(0)$ & \\
\hline \multicolumn{4}{|l|}{ NYHA class, n (\%) } \\
\hline I & $28(28.9)$ & $30(35.3)$ & .868 \\
\hline II & $56(57.7)$ & $49(57.6)$ & \\
\hline III & $8(8.2)$ & $6(7.1)$ & \\
\hline IV & $5(5.2)$ & $6(6.6)$ & \\
\hline \multicolumn{4}{|l|}{ Underlying diseases, n (\%) } \\
\hline Gastrointestinal disease & $6(6.1)$ & $3(4.3)$ & .501 \\
\hline Hypertension & $21(21.6)$ & $16(17.6)$ & .483 \\
\hline Diabetes & $11(11.3)$ & $13(14.3)$ & .545 \\
\hline Hyperlipidemia & $9(9.3)$ & $6(6.6)$ & .497 \\
\hline $\mathrm{CHD}$ & $25(25.8)$ & $16(17.6)$ & .174 \\
\hline Stroke & $2(2.1)$ & $1(1.1)$ & 1.000 \\
\hline Active smoking & $25(25.8)$ & $19(20.9)$ & .428 \\
\hline >Alcoholism & $0(0)$ & $1(1.1)$ & .484 \\
\hline EuroSCORE II & $6.2 \pm 2.3$ & $6.5 \pm 2.5$ & .329 \\
\hline GICS score & $12.5 \pm 6.4$ & $13.1 \pm 6.0$ & .654 \\
\hline \multicolumn{4}{|l|}{$\begin{array}{l}\text { Conventional coagulation } \\
\text { function test }\end{array}$} \\
\hline PT, s & $13.4 \pm 3.2$ & $12.8 \pm 3.9$ & .473 \\
\hline INR & $1.06 \pm 0.4$ & $1.10 \pm 0.8$ & .516 \\
\hline APTT, s & $38.9 \pm 7.2$ & $36.6 \pm 6.0$ & .210 \\
\hline TT, s & $17.2 \pm 4.5$ & $18.3 \pm 6.3$ & .398 \\
\hline
\end{tabular}

Table 1. Clinical Data (cont.)

\begin{tabular}{lccc}
\hline & $\begin{array}{c}\text { Group A } \\
(\mathrm{n}=97)\end{array}$ & $\begin{array}{c}\text { Group B } \\
(\mathrm{n}=91)\end{array}$ & $P$ \\
\hline Fib, g/L & $3.2 \pm 1.9$ & $3.1 \pm 2.8$ & .429 \\
TEG & & & \\
R, s & $6.6 \pm 4.1$ & $7.1 \pm 5.3$ & .280 \\
K, s & $1.9 \pm 0.6$ & $2.1 \pm 0.8$ & .578 \\
Angle (degree) & $60.2 \pm 12.2$ & $59.1 \pm 7.9$ & .372 \\
MA, mm & $55.9 \pm 9.2$ & $60.2 \pm 10.2$ & .121 \\
LY30, \% & $2.1 \pm 2.2$ & $2.9 \pm 3.1$ & .482 \\
EPL, \% & $1.3 \pm 1.0$ & $1.9 \pm 1.3$ & .552 \\
Routine blood test & & & \\
WBC, $\times 10^{9} / \mathrm{L}$ & $8.9 \pm 6.7$ & $9.8 \pm 7.1$ & .413 \\
Hb, g/L & $121.2 \pm 10.1$ & $117.6 \pm 9.9$ & .370 \\
PLT, $\times 10^{9} / \mathrm{L}$ & $201.9 \pm 29.2$ & $223.2 \pm 36.7$ & .292 \\
\hline
\end{tabular}

The primary analyses revealed that there were no significant differences between the patients in terms of general clinical features. CVD indicates cardiac valvular disease; CAD, coronary artery disease; $\mathrm{CHD}$, congenital heart disease; $\mathrm{HT}$, heart transplantation; $\mathrm{AAD}$, acute aortic dissection; BMI, body mass index; NYHA, New York heart association; PT, prothrombin time; INR, international normalized ratio; APTT, activated partial prothrombin time; TT, thrombin time; Fib, fibrinogen; TEG, thromboelastography; WBC, white blood cell; Hb, hemoglobin; PLT, platelet.

The strategy of a continuous intravenous infusion of PPI combined with early enteral nutrition was applied in 97 of the included patients (Group A, 97/188, 51.6\%), and the strategy of a bolus intravenous infusion of PPI combined with late enteral nutrition was applied in the patients of a second group (Group B, 91/188, 48.4\%). The primary analyses revealed that there were no significant differences between the patients in terms of protopathy, age, sex, $\mathrm{BMI}$, drug history of anticoagulants or antiplatelet agents, coagulation function, the Child-Pugh score, NYHA class, underlying diseases including history of gastrointestinal disease, hypertension, diabetes, hyperlipidemia, CAD, stroke, active smoking, alcoholism, GICS score and EuroSCORE II (Table 1).

\section{Surgical and Perioperative Treatments}

The chi-square test revealed that there were no significant differences in the types of surgical correction between the patients in the two groups. Wilcoxon rank sum tests also indicated that the duration of surgery, $\mathrm{CPB}$, aortic crossclamping, HCA, thoracic closure and perioperational blood loss had no significant differences in the patients in these two groups (Table 2).

\section{Laboratory Examination}

Wilcoxon rank sum tests revealed that there were no significant differences in the results of the preoperative and 
Table 2. Surgical and Intraoperative Treatments

\begin{tabular}{lccc}
\hline & $\begin{array}{c}\text { Group A } \\
(\mathrm{n}=97)\end{array}$ & $\begin{array}{c}\text { Group B } \\
(\mathrm{n}=91)\end{array}$ & $P$ \\
\hline Surgery, min & $201.7 \pm 256.9$ & $223.0 \pm 199.7$ & .089 \\
CPB, min & $61.5 \pm 121.8$ & $70.8 \pm 155.2$ & .101 \\
Aortic cross-clamping, min & $38.8 \pm 89.1$ & $45.1 \pm 109.7$ & .091 \\
HCA, min & $9(9.3)$ & $8(8.8)$ & .907 \\
Blood loss, mL & $504.9 \pm 109.6$ & $550.9 \pm 205.8$ & .087 \\
Allogenetic transfusion & & & \\
$\quad$ RBC, $U$ & $2.8 \pm 7.9$ & $3.0 \pm 10.1$ & .452 \\
$\quad$ FFP, mL & $300.4 \pm 56.3$ & $347.1 \pm 79.3$ & .088 \\
PLT, $U$ & $1.2 \pm 2.4$ & $1.5 \pm 2.9$ & .381 \\
CP, $U$ & $2.3 \pm 5.5$ & $2.9 \pm 6.9$ & .210 \\
\hline
\end{tabular}

Pearson chi-square test and Wilcoxon rank sum test revealed that there were no significant differences in the surgical and intraoperative treatments between the patients in these two groups. CPB indicates cardiopulmonary bypass; HCA, hypothermic circulatory arrest; RBC, red blood cell; FFP, fresh frozen plasma; PLT, platelet; CP, cryoprecipitation.

postoperative routine blood tests, conventional coagulation function test, and TEG between the two groups (Table 3).

\section{Postoperative Statistics}

Wilcoxon rank sum tests showed that the period of time from the end of surgery to eating for the first time for the patients of group A was significantly shorter than for the patients in group B $(14.5 \pm 5.9 \mathrm{~h}$ versus $20.9 \pm 8.9, P=.001)$. However, we could not discover significant differences in the length of time from the end of surgery to defecation for the first time $(29.1 \pm 8.1 \mathrm{~h}$ versus $35.2 \pm 10.1, P=.054)$. We also discovered no significant differences in the incidences of postoperative meteorism, nausea, vomiting, abdominal pain, diarrhea, constipation, headache and the result of the fecal OB test between the two groups. The incidence of gastrointestinal hemorrhage in all patients was $5.9 \%(11 / 188)$ and the incidences of gastrointestinal hemorrhage $(1 / 97,1.0 \%$ versus $10 / 91,11.0 \%$, $P=.004)$ and hematemesis $(1 / 97,1.0 \%$ versus $7 / 91,7.7 \%$, $P=.030)$ in the patients in group A were significantly higher than the incidences in group B. However, no significant difference was found in the rate of hematochezia (1/97, 1.0\% versus $5 / 91,5.5 \%, P=.109)$ between the two groups.

No significant differences in the prescribing rates of vasoactive agent, anticoagulants, antiplatelet agents and high-dose glucocorticoids were identified between the patients in these two groups. The rates of the use of IABP and/or ECMO were also confirmed to be similar between the two groups. The 30 -day postoperative mortality in all the patients was 3.2\% (6/188). The mortality in the patients of group A seemed to be lower than that in the patients of group B $(1 / 97,1.0 \%$ versus $5 / 91,5.5 \%, P=.082$ ), but we could not see any significant differences between the two groups. Moreover, the duration of postoperative mechanical ventilation, ICU stay and

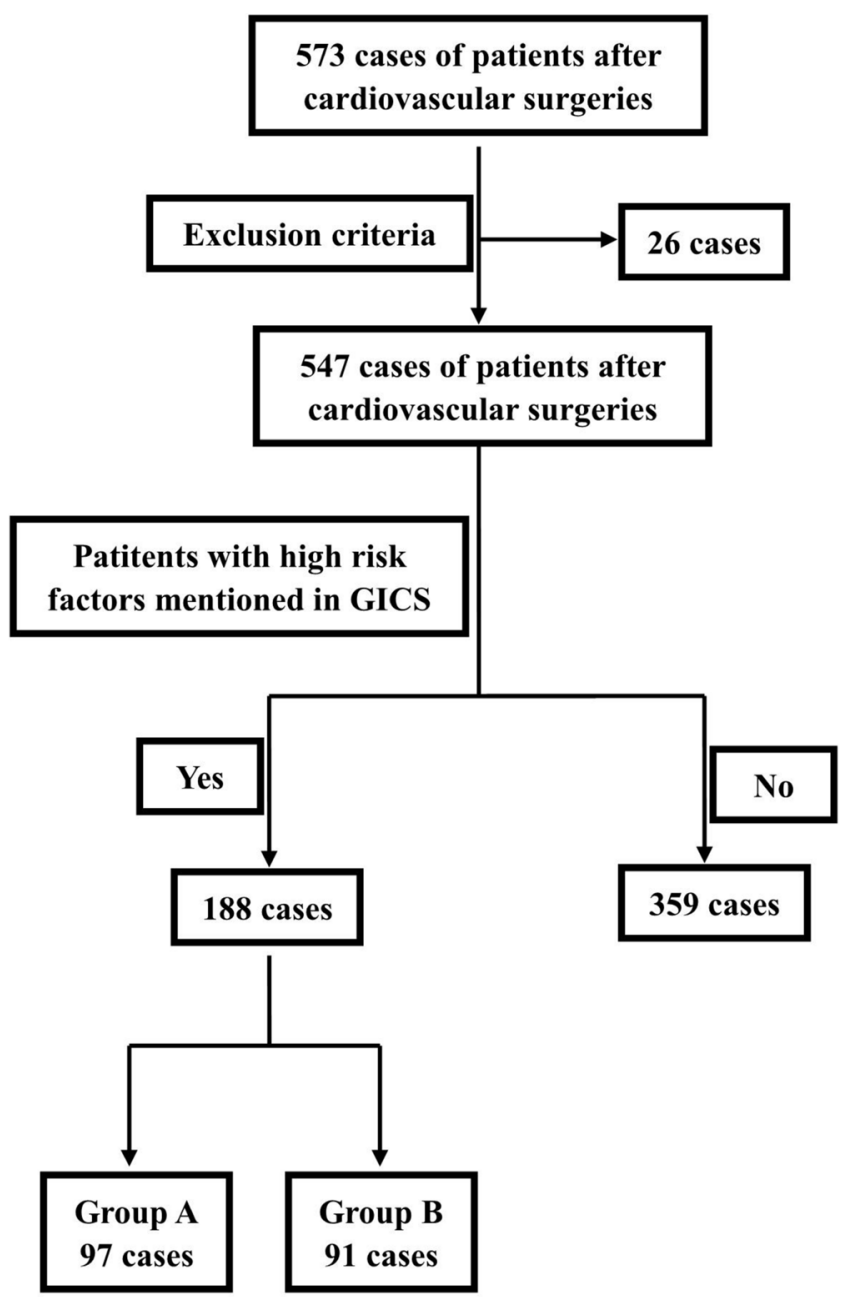

Figure 1. This study included 573 patients who underwent cardiovascular surgeries. Twenty-six patients who met the exclusion criteria were ruled out, and 188 cases that were consistent with at least one of the high-risk factors mentioned in the GICS score were divided into two groups according to the prophylactic strategy of gastrointestinal hemorrhage.

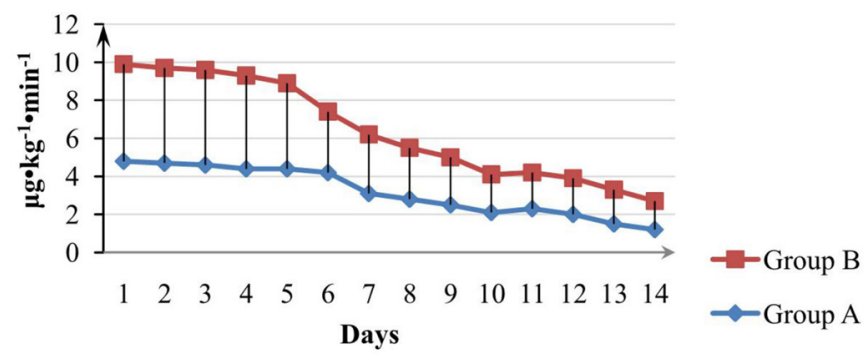

Figure 2. Repeated measures analysis revealed that the dosage of the postoperative dopamine of the patients in the two groups did not show significant differences within the 14 days after surgery $(P=.578)$.

hospitalization were comparable, and we could not find any significant differences in the incidences of postoperative complications, excluding gastrointestinal hemorrhage between the two groups (Table 3; Figures 2, 3, 4, 5). 


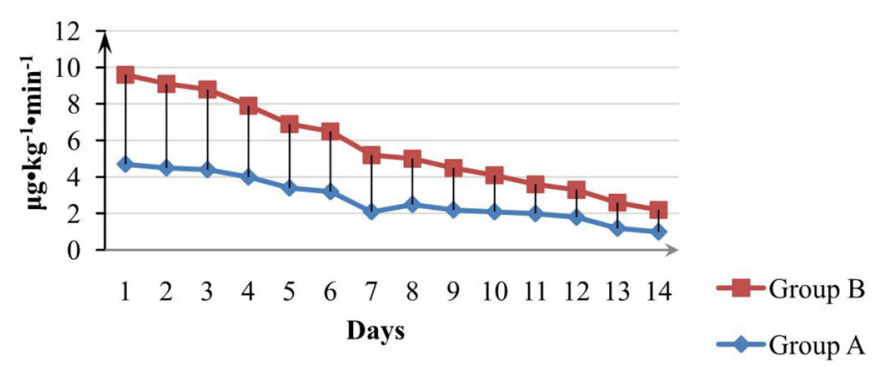

Figure 3. Repeated measures analysis revealed that the dosage of the postoperative dobutamine of the patients in the two groups did not show significant differences within the 14 days after surgery $(P=.662)$.

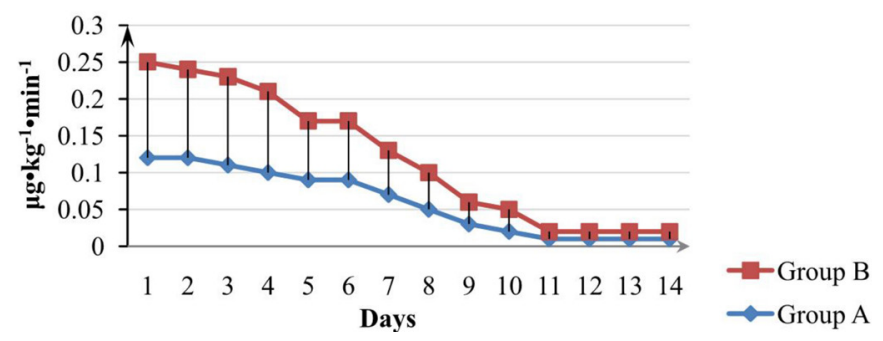

Figure 4. Repeated measures analysis revealed that the dosage of the postoperative adrenaline of the patients in the two groups did not show significant differences within the 14 days after surgery $(P=.409)$.

\section{Analysis of Patients with Postoperative Gastrointestinal Hemorrbage}

Eleven patients with postoperative gastrointestinal hemorrhage $(11 / 188,5.9 \%)$ were observed in this research, and six of them $(6 / 11,54.4 \%)$ died within 30 days after their surgeries. The main causes of death were MODS (1 case from the patients in group A and 3 cases from the patients in group B) and LCOT (2 cases from patients in group B) (Table 4).

\section{Survival Curves}

Survival curve analyses based on 15 months of observation revealed no significant difference in the survival rate or the median survival time between the patients in group $\mathrm{A}$ and group B (Figure 6).

\section{DISCUSSION}

Gastrointestinal hemorrhage after cardiovascular surgery can be fatal and considerably difficult to diagnose early because of postoperative sedation, analgesia and mechanical ventilation. Thus, it is crucial for cardiovascular surgeons and physicians in the ICU to find an optimal therapeutic strategy to prevent gastrointestinal hemorrhage in high-risk patients after cardiovascular surgery. Currently, bolus intravenous infusion of PPI is a conventional prophylactic strategy for gastrointestinal hemorrhage after cardiovascular surgery [Madsen 2014]. However, in our research, $11 \%$ of patients suffered from postoperative gastrointestinal hemorrhage, and $50 \%$ of them died. Therefore, it is important to find a comprehensive therapy that would

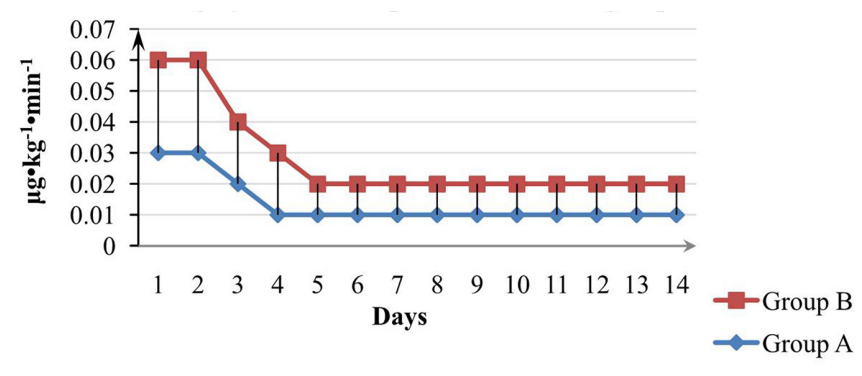

Figure 5. Repeated measures analysis revealed that the dosage of the postoperative noradrenaline of the patients in the two groups did not show significant differences within the 14 days after surgery $(P=.781)$.

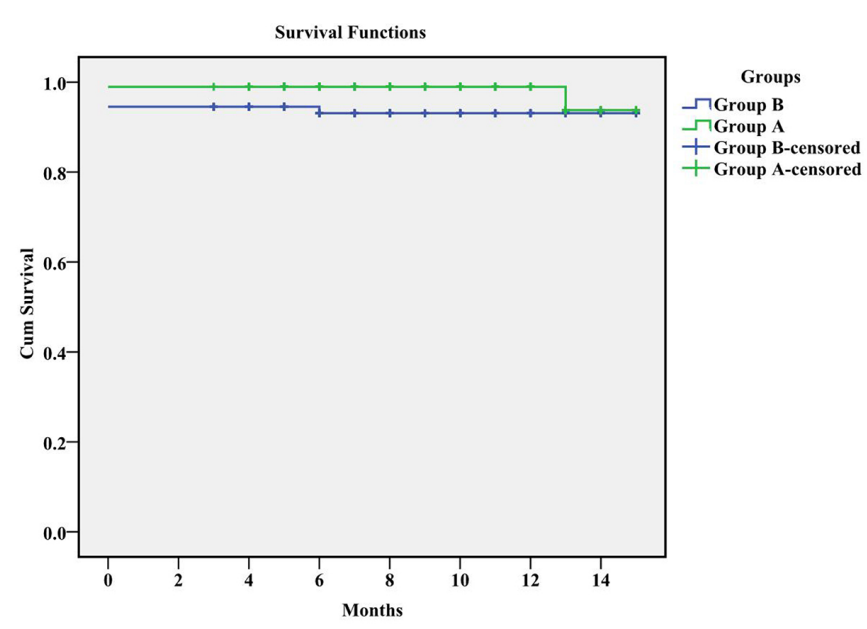

Figure 6. Kaplan-Meier plots reveal that there were no significant differences in the survival rates or the median survival times between the patients in group $\mathrm{A}$ and the patients in group B (log-rank result: $\chi^{2}=$ $2.480, P=.115)$, (14.7 versus 14.0 months).

effectively prevent gastrointestinal hemorrhage after cardiovascular surgery and improve the outcomes of these patients.

Antacid agents used to play a key role in the empirical treatment to prevent gastrointestinal hemorrhage after cardiovascular surgery. However, certain questions need to be answered, including those concerned with choosing the dosage of the antacid agent and the timing of administration. According to our past experience, the strategy of using a bolus intravenous infusion of PPI did not effectively reduce the incidence of gastrointestinal hemorrhage after cardiovascular surgery. Thus, we improved the conventional treatment so that patients were administered PPI through continuous intravenous injection ( $80 \mathrm{mg}$ qd) within 3-7 days after surgery, and PPI withdrawal was initiated when the hemodynamics of patients were stable after extubation and they could eat normally or be fed with enteral nutrition through a nasogastric tube without gastrointestinal symptoms such as abdominal pain, ventosity or dysphoria. The $\mathrm{pH}$ value of gastric mucosa could be continuously maintained above 6.0 with this modified antacid strategy without rebounding during the interval of bolus administration of PPI [Chwiesko 2016]. 
Table 3. Postoperative Statistics

\begin{tabular}{|c|c|c|c|}
\hline & $\begin{array}{l}\text { Group A } \\
(n=97)\end{array}$ & $\begin{array}{l}\text { Group B } \\
(n=91)\end{array}$ & $P$ \\
\hline Eating for the first time, $h$ & $14.5 \pm 5.9$ & $20.9 \pm 8.9$ & .001 \\
\hline Defecation for the first time, $\mathrm{h}$ & $29.1 \pm 8.1$ & $35.2 \pm 10.1$ & .054 \\
\hline \multicolumn{4}{|l|}{ Gastrointestinal symptoms, n (\%) } \\
\hline Hematemesis & $1(1.0)$ & $7(7.7)$ & .030 \\
\hline Hematochezia & $1(1.0)$ & $5(5.5)$ & .109 \\
\hline Gastrointestinal hemorrhage & $1(1.0)$ & $10(11.0)$ & .004 \\
\hline Meteorism & $5(5.2)$ & $5(5.5)$ & .917 \\
\hline Nausea or vomiting & $9(9.3)$ & $11(12.1)$ & .532 \\
\hline Abdominal pain & $4(4.1)$ & $2(2.2)$ & .683 \\
\hline Hematochezia & $7(7.2)$ & $6(6.6)$ & .866 \\
\hline Constipation & $10(10.3)$ & $12(13.2)$ & .540 \\
\hline $\begin{array}{l}\text { Gastrointestinal hemorrhage for } \\
\text { the first time, } d\end{array}$ & $4.6 \pm 3.4$ & $5.2 \pm 4.9$ & .361 \\
\hline Postoperative thoracic drainage, $\mathrm{mL}$ & $892.2 \pm 309.3$ & $948.3 \pm 403.1$ & .101 \\
\hline \multicolumn{4}{|l|}{ Anticoagulants, n (\%) } \\
\hline Warfarin & $65(67.0)$ & $64(70.3)$ & .624 \\
\hline Heparin & $59(60.8)$ & $55(60.4)$ & .957 \\
\hline Antiplatelet drug, n (\%) & $20(20.6)$ & $18(19.8)$ & .886 \\
\hline high-dose glucocorticoids, n (\%) & $6(6.2)$ & $9(9.9)$ & .349 \\
\hline Hypoproteinemia, n (\%) & $12(12.4)$ & $16(17.6)$ & .316 \\
\hline \multicolumn{4}{|l|}{ Mechanical assistance, n (\%) } \\
\hline IABP & $4(4.1)$ & $3(3.3)$ & .765 \\
\hline ECMO & $1(1.0)$ & $2(2.2)$ & .611 \\
\hline Mechanical ventilation, $\mathrm{h}$ & $15.9 \pm 25.4$ & $16.4 \pm 28.5$ & .717 \\
\hline ICU stay, $d$ & $4.3 \pm 11.2$ & $5.6 \pm 15.5$ & .538 \\
\hline Hospitalization, d & $11.2 \pm 19.4$ & $13.1 \pm 24.3$ & .228 \\
\hline 30-day mortality, n (\%) & $1(1.0)$ & $5(5.5)$ & .082 \\
\hline \multicolumn{4}{|l|}{ Postoperative complications, n (\%) } \\
\hline LCOT & $4(4.1)$ & $4(4.4)$ & .926 \\
\hline $\mathrm{AKI}$ requiring dialysis & $8(8.2)$ & $9(9.2)$ & .695 \\
\hline Child-Pugh score 3-4 & $7(7.2)$ & $8(8.8)$ & .690 \\
\hline Sepsis & $4(4.1)$ & $6(6.6)$ & .451 \\
\hline MODS & $2(2.1)$ & $6(6.6)$ & .159 \\
\hline ARDS & $3(3.1)$ & $3(3.3)$ & .937 \\
\hline Pulmonary infection & $12(12.4)$ & $18(19.8)$ & .166 \\
\hline Stroke & $2(2.1)$ & $2(2.2)$ & 1.000 \\
\hline Reoperation for bleeding & $2(2.1)$ & $1(1.1)$ & .599 \\
\hline Hypotension & $7(7.2)$ & $10(11.0)$ & .367 \\
\hline $\mathrm{Lac}>3 \mathrm{mmol} / \mathrm{L}$ & $15(15.5)$ & $18(19.8)$ & .437 \\
\hline \multicolumn{4}{|l|}{ Allogenetic transfusion } \\
\hline$R B C, U$ & $2.9 \pm 4.2$ & $4.5 \pm 5.9$ & .065 \\
\hline
\end{tabular}

Table 3. Postoperative Statistics (cont.)

\begin{tabular}{|c|c|c|c|}
\hline & $\begin{array}{l}\text { Group A } \\
(n=97)\end{array}$ & $\begin{array}{l}\text { Group B } \\
(n=91)\end{array}$ & $P$ \\
\hline FFP, mL & $302.3 \pm 102.5$ & $494 \pm 192.7$ & .071 \\
\hline PLT, U & $0.8 \pm 0.5$ & $1.1 \pm 0.6$ & .075 \\
\hline $\mathrm{CP}, \mathrm{U}$ & $1.2 \pm 2.2$ & $2.0 \pm 2.8$ & .093 \\
\hline \multicolumn{4}{|l|}{ Routine blood test } \\
\hline WBC, $\times 10^{9} / \mathrm{L}$ & $12.5 \pm 5.3$ & $12.9 \pm 6.1$ & .478 \\
\hline $\mathrm{Hb}, \mathrm{g} / \mathrm{L}$ & $11.0 \pm 3.1$ & $9.8 \pm 5.7$ & .145 \\
\hline $\mathrm{PLT}, \times 10^{9} / \mathrm{L}$ & $159.6 \pm 20.5$ & $144.4 \pm 39.3$ & .238 \\
\hline \multicolumn{4}{|c|}{ Conventional coagulation function test } \\
\hline PT, s & $14.3 \pm 3.8$ & $13.1 \pm 4.9$ & .088 \\
\hline INR & $1.09 \pm 0.5$ & $1.12 \pm 1.0$ & .211 \\
\hline APTT, s & $42.3 \pm 6.5$ & $46.6 \pm 5.7$ & .322 \\
\hline TT, s & $18.3 \pm 4.0$ & $18.5 \pm 5.8$ & .836 \\
\hline Fib, g/L & $3.8 \pm 2.1$ & $3.0 \pm 3.8$ & .559 \\
\hline \multicolumn{4}{|l|}{ TEG } \\
\hline $\mathrm{R}, \mathrm{s}$ & $6.7 \pm 5.5$ & $7.3 \pm 4.6$ & .184 \\
\hline $\mathrm{K}, \mathrm{s}$ & $2.0 \pm 0.5$ & $2.0 \pm 0.6$ & 1.000 \\
\hline Angle (degree) & $63.6 \pm 15.3$ & $66.9 \pm 8.3$ & .112 \\
\hline $\mathrm{MA}, \mathrm{mm}$ & $59.2 \pm 8.7$ & $62.6 \pm 9.1$ & .248 \\
\hline LY30, \% & $1.4 \pm 1.2$ & $1.9 \pm 2.1$ & .234 \\
\hline EPL, \% & $1.2 \pm 0.8$ & $1.4 \pm 1.0$ & .109 \\
\hline
\end{tabular}

Wilcoxon rank sum tests showed that the length from the end of the operation to eating for the first time in the patients of group A was significantly shorter than the patients of group B. The incidences of gastrointestinal hemorrhage and hematemesis of the patients in group A were significantly higher than those in group B. The 30-day mortality in the patients of group A seemed to be lower than the patients of group $B$. IABP indicates intraaortic balloon pump; ECMO, extracorporeal membrane oxygenation; LCOT, low cardiac output syndrome; AKI, acute kidney injury; MODS, multiple organ dysfunction syndrome; ARDS, acute respiratory distress syndrome; Lac, lactic acid; RBC, red blood cell; FFP, fresh frozen plasma; PLT, platelet; CP, cryoprecipitation; WBC, white blood cell; Hb, hemoglobin; PT, prothrombin time; INR, international normalized ratio; APTT, activated partial prothrombin time; TT, thrombin time; Fib, fibrinogen; TEG, thrombelastogram.

Due to the complex pathogenic conditions of the patients after cardiovascular surgery, the administration of PPI alone is not sufficient to prevent gastrointestinal hemorrhage. Strategies for early eating have been reported to promote the recovery of gastrointestinal function through early enteral nutrition. Moreover, a lower incidence of intestinal flora imbalance has been observed in some studies with supplementation of intestinal probiotics after surgery [Marik 2010; Lee 2014; Lewis 2009]. In view of this, postoperative patients were fed probiotics and enteral nutrition through a nasogastric tube or by oral administration within 12-24 h after surgery, regardless of extubation. Wilcoxon rank sum tests 
Table 4. Analysis of Patients with Postoperative Gastrointestinal Hemorrhage

\begin{tabular}{|c|c|c|c|}
\hline & $\begin{array}{c}\text { Group A } \\
(n=1)\end{array}$ & $\begin{array}{l}\text { Group B } \\
(n=10)\end{array}$ & $P$ \\
\hline \multicolumn{4}{|l|}{ Protopathy, n (\%) } \\
\hline CAD & $1(100)$ & $2(20.0)$ & .273 \\
\hline$A A D$ & $0(0)$ & $3(30.0)$ & 1.000 \\
\hline CVD & $0(0)$ & $5(50.0)$ & 1.000 \\
\hline \multicolumn{4}{|l|}{ Cause of death, $\mathrm{n}(\%)$} \\
\hline MODS & $1(100)$ & $3(30.0)$ & .364 \\
\hline LCOT & $0(0)$ & $2(20.0)$ & 1.000 \\
\hline 30-day mortality, n (\%) & $1(100)$ & $5(50.0)$ & 1.000 \\
\hline \multicolumn{4}{|l|}{ Complications, n (\%) } \\
\hline CPR & $0(0)$ & $1(10.0)$ & 1.000 \\
\hline IABP & $1(100)$ & $1(10.0)$ & .182 \\
\hline LCOT & $1(100)$ & $2(20.0)$ & .273 \\
\hline ECMO & $0(0)$ & $2(20.0)$ & 1.000 \\
\hline AKI requiring dialysis & $1(100)$ & $2(20.0)$ & .273 \\
\hline Coagulopathy & $0(0)$ & $1(10.0)$ & 1.000 \\
\hline Child-Pugh score $>2$ & $1(100)$ & $1(10.0)$ & .182 \\
\hline Sepsis & $0(0)$ & $2(20.0)$ & 1.000 \\
\hline MODS & $1(100)$ & $5(50.0)$ & 1.000 \\
\hline ARDS & $0(0)$ & $1(10.0)$ & 1.000 \\
\hline Pulmonary infection & $1(100)$ & $5(50.0)$ & 1.000 \\
\hline Stroke & $0(0)$ & $1(10.0)$ & 1.000 \\
\hline
\end{tabular}

Eleven patients with postoperative gastrointestinal hemorrhage (11/188, $5.9 \%)$ were observed in this research, and 6 of them $(6 / 11,54.4 \%)$ were dead within 30 days after the operation. The main causes of deaths were MODS ( 1 case from patients in group $A$ and 3 cases from patients in group B) and LCOT ( 2 cases from patients in group $B$ ). CAD indicates coronary artery disease; AAD, acute aortic dissection; CVD, cardiac valvular disease; MODS, multiple organ dysfunction syndrome; LCOT, low cardiac output syndrome; IABP, intra-aortic balloon pump; ECMO, extracorporeal membrane oxygenation; AKI, acute kidney injury; ARDS, acute respiratory distress syndrome.

revealed that the length of time from the end of the operation to eating for the first time significantly decreased from $20.9 \pm$ $8.9 \mathrm{~h}$ (group B) to $14.5 \pm 5.9 \mathrm{~h}$ (group A).

It is worth noting that this new prophylactic treatment required coordination with our anesthesiologists, and shortacting sedation, muscle relaxants and analgesics should be used to accelerate the recovery of postoperative gastrointestinal function. Patients should be closely monitored and enteral nutrition should be stopped in the presence of ventosity, nausea, vomiting, abnormal borborygmus or an increase in gastric juices (cumulative volume $>200 \mathrm{~mL}$ ).

Using an inappropriate enteral nutrition strategy must be avoided for fear of aggravating the progress of postoperative recovery of the gastrointestinal function. Ten patients with gastrointestinal symptoms were observed in group A. This included six patients who were considered to be suffering from the residual effects of anesthesia and who recovered after symptomatic treatment; three patients who were considered to have gastrointestinal congestion induced by cardiac insufficiency and continued to receive enteral nutrition after the improvement of cardiac function; and one patient in whom the volume of nasogastric tube feeding was reduced due to postoperative MODS but who died within 10 days after surgery. All patients with postoperative gastrointestinal complications ultimately recovered and were discharged, excluding the one patient who died from MODS.

The analysis of clinical data revealed that there were no significant differences in the preoperative pathogenetic condition, protopathy, surgical and perioperative treatments, pharmacotherapy and postoperative complications. Therefore, we believed that the incidence of gastrointestinal hemorrhage could be effectively reduced by the new prophylactic strategy. Moreover, patients in group A showed lower 30-day postoperative mortality. However, no significant differences were found between the two groups, which could be explained by the small sample size. A similar phenomenon has been observed in the volume of perioperative transfusion between the patients in these two groups.

Interestingly, we did not find significant differences in the incidence of hematochezia in the patients in these two groups. However, hematemesis was significantly reduced in the patients in group A. This demonstrated that the new prophylactic strategy only maintained the integrity of the mucous bicarbonate barrier in the gastric mucosa and was inclined to protect the integrity of the upper gastrointestinal mucosa rather than lower gastrointestinal mucosa. In view of the limitations of this new prophylactic strategy, a more effective comprehensive treatment for the prevention of gastrointestinal hemorrhage should be the next goal of additional studies.

The major limitation of this study is the relatively small number of patients in whom gastrointestinal complications developed. Further validation requires a multicenter, randomized controlled study with a large sample size and longterm follow up.

\section{Conclusion}

Continuous intravenous infusion of PPI combined with early enteral nutrition could effectively prevent gastrointestinal hemorrhage and reduce 30-day mortality after cardiovascular surgery in high-risk patients. However, the new prophylactic strategy was inclined to protect the integrity of the upper gastrointestinal mucosa rather than that of the lower gastrointestinal mucosa. Further validation requires longterm follow-up and a multicenter, randomized controlled study with a large sample size.

\section{REFERENCES}

Ait Houssa M, Selkane C, Moutaki Allah Y, et al. 2007. Upper digestive bleedings after cardiac surgery. Ann Cardiol Angeiol (Paris). 56:126-9. 
Andersson B, Nilsson J, Brandt J, et al. 2005. Gastrointestinal complications after cardiac surgery. Br J Surg 92:326-33.

Andersson B, Andersson R, Brandt J, Höglund P, Algotsson L, Nilsson J. 2010. Gastrointestinal complications after cardiac surgery - improved risk stratification using a new scoring model. Interact Cardiovasc Thorac Surg 10:366-70.

Chaudhry R, Zaki J, Wegner R, et al. 2017. Gastrointestinal complications after cardiac surgery: A nationwide population-based analysis of morbidity and mortality predictors. J Cardiothorac Vasc Anesth 31:1268-74.

Chwiesko A, Charkiewicz R, Niklinski J, et al. 2016. Effects of different omeprazole dosing on gastric $\mathrm{pH}$ in non-variceal upper gastrointestinal bleeding: A randomized prospective study. J Dig Dis 17:588-99.

D'Ancona G, Baillot R, Poirier B, et al. 2003. Determinants of gastrointestinal complications in cardiac surgery. Tex Heart Inst J 30:280-5.

Fan H, Zheng Z, Feng W, et al. 2010. Risk factors and prevention of upper gastrointestinal hemorrhage after a coronary artery bypass grafting operation. Surg Today 40:931-5

Grus T, Rohn V, Brlicová L, et al. 2014. Gastrointestinal complications after cardiac surgery: eight years experience in a single center. Acta Chir Belg 114:332-7.

Krawiec F, Maitland A, Duan Q, Faris P, Belletrutti PJ, Kent WDT. 2017. Duodenal ulcers are a major cause of gastrointestinal bleeding after cardiac surgery. J Thorac Cardiovasc Surg 154:181-8.

Lee SH, Jang JY, Kim HW, et al. 2014. Effects of early enteral nutrition on patients after emergency gastrointestinal surgery: a propensity score matching analysis. Medicine 93:e323.

Lewis SJ, Andersen HK, Thomas S. 2009. Early enteral nutrition within $24 \mathrm{~h}$ of intestinal surgery versus later commencement of feeding: a systematic review and meta-analysis. J Gastrointest Surg 13:569-75.

Madsen KR, Lorentzen K, Clausen N, et al. 2014. Danish Society of Intensive Care Medicine; Danish Society of Anesthesiology and Intensive Care Medicine. Guideline for stress ulcer prophylaxis in the intensive care unit. Dan Med J 61:C4811.

Marik PE, Vasu T, Hirani A, et al. 2010. Stress ulcer prophylaxis in the new millennium: a systematic review and meta-analysis. Crit Care Med 38:2222-8.

Ohri SK, Velissaris T. 2006. Gastrointestinal dysfunction following cardiac surgery. Perfusion 21:215-23.

Patel AJ, Som R. 2013. What is the optimum prophylaxis against gastrointestinal haemorrhage for patients undergoing adult cardiac surgery: histamine receptor antagonists, or proton-pump inhibitors? Interact Cardiovasc Thorac Surg. 16:356-60.

Perugini RA, Orr RK, Porter D, et al. 1997. Gastrointestinal complications following cardiac surgery. An analysis of 1477 cardiac surgery patients. Arch Surg 132:352-7.

Quenot JP, Thiery N, Barbar S. 2009. When should stress ulcer prophylaxis be used in the ICU? Curr Opin Crit Care 15:139-43.

Rodriguez R, Robich MP, Plate JF, et al. 2010. Gastrointestinal complications following cardiac surgery: a comprehensive review. J Card Surg 25:188-97. 